Die Bedeutung von Produkt-Ökobilanzen in der staatlichen Umweltpolitik

\title{
Instrument der \\ Entscheidungsfindung
}

\section{Anhand von vier Thesen diskutiert Harald Neitzel die Bedeutung von Produkt- Ökobilanzen in der stautlichen Umweltpolitik (1). Er betont dabei unter ande- rem, daß die ökologische Verbesserung von Produkten eine gemeinsame Auf- gabe von Wirtschaft, Staat und Gesellschaft ist.}

\section{Von Harald Neitzel} hese 1: Ökobilanzen mit ihrer Vergleichs-, Optimierungs- und Kommunikationsfunktion sind ein ,ökologisches“ Instrument der umweltpolitischen Entscheidungsfindung. Ökonomische und soziale Faktoren - auch im Sinne des Leitbildes der Nachhaltigkeit - werden in Ökobilanzen weitgehend ausgegrenzt. Dies kann zu Zielkonflikten auch in der Umweltpolitik führen.

Die Berücksichtigung von Ökobilanzen in umweltpolitischen Entscheidungsprozessen ist in der internationalen Ökobilanz-Norm allgemein anerkannt worden („public policy making“) (2). Konsensfähige und wirksame staatliche Umweltpolitik muß sich über die Ressortgrenzen im engeren Sinne hinaus um eine intensive Integration in andere Fachpolitiken wie zum Beispiel die Verkehrs-, Landwirtschafts- und Wirtschaftspolitik bemühen. Im Vergleich zu Ökobilanzen muß sie daher sehr viel stärker die ökonomischen und sozialen Auswirkungen von Handlungsalternativen einbeziehen. Deutlich wird dies am Beispiel der Umsetzung der Ökobilanz Rapsöl (3). Trotz gegenteiliger Ökobilanzergebnisse wird die Förderung des sogenannten „Bio-Diesels" politisch in Bund und Ländern weiterhin überwiegend befürwortet. Die positiven sozialen und ökonomischen Auswirkungen - Wertschöpfungen im Inland zu Lasten von Importen erscheinen zu offenkundig. Dies zeigt zugleich, daß es keinerlei Automatismus zwischen staatlich initiierten Ökobilanzprojekten und (umwelt-) politisch zu ziehenden Schlußfolgerungen gibt.

\section{Beilagenhinweis}

Dieser Ausgabe liegen Informationen des Rhombos-Verlages bei. Wir bitten um freundliche Beachtung.
These 2: Die ökologische Verbesserung der Produkte mittels ökobilanzen ist eine gemeinsame Aufgabe von Wirtschaft, Staat und Gesellschaft. Dies kann nur im Wege eines fruchtbaren Dialoges und der Achtung der jeweiligen Interessen gelingen. Zu Recht wird in der ISO 14040 als wichtigste Anwendung die Funktion von Ökobilanzen als eine Entscheidungsgrundlage für die ökologische Verbesserung der Produkte (,improvement") angefiihrt. Dies drückt sich z. B. durch die weitaus überwiegende Zahl der wirtschaftsseitig initiierten Ökobilanzprojekte (Unternehmen, Unternehmensverbünde und Verbände) aus (4). Bei dieser Würdigung darf allerdings nicht die Rolle des Staates und der gesellschaftlichen Gruppen vergessen werden.

Aufgabe des Staates ist es hierbei vor allem, maßgebliche Umweltqualitäts- und Umwelthandlungsziele festzulegen und hieraus abgeleitete Prioritäten ökologisch orientierter Produktentwicklung $\mathrm{zu}$ benennen und $\mathrm{zu}$ begründen und diese mit allen beteiligten Kreisen zu diskutieren. Dieser Diskussionsprozeß stellt auch eine Brücke zu der anhaltenden Diskussion um eine Lösung der Bewertungsprobleme in Ökobilanzen dar, die nur unter Einbeziehung von Prioritäten im Umweltschutz gelingen kann (5). Zugleich kann der Staat, insbesondere auch angesichts der zunehmenden Bedeutung freiwilliger Maßnahmen bei Vorsorgezielsetzungen, nicht aus der Verantwortung entlassen werden, von sich aus Initiativen zu ergreifen, um die - auch mittels Ökobilanzen als richtig erkannten - Zielsetzungen der Umweltpolitik auch im Produktbereich $\mathrm{zu}$ verfolgen und $\mathrm{zu}$ beschleunigen.

In diesem Sinne ist auch die Beteiligung der gesellschaftlichen Gruppen, insbesondere der Umwelt- und Verbraucherverbände und der Gewerkschaften, an der Ökobilanzdiskussion eine notwendige Voraussetzung, um die Ziele der produktbezogenen Umweltpolitik zu erreichen. Zum einen sind diese - verwiesen wird auf die anhaltende Diskussion um eine nachhaltige Entwicklung - nur im breiten gesellschaftlichen Konsens tragfähig. Die gesellschaftlichen Gruppen sind dabei für die Akzeptanz in der Bevölkerung von maßgeblicher Bedeutung. Zum anderen - auch das signalisieren Ökobilanzen wie z. B. die Produktlinienanalyse Waschen und Waschmittel (6) - sind Umweltziele im Produktbereich häufig nur dann erreichbar, wenn auch die Verbraucher ihren Part bei der Nutzung und der Entsorgung dazu beitragen.

These 3: Bei den Anwendungen von Ökobilanzen liegen sowohl in der Umweltpolitik als auch in den Unternehmen erste Erfahrungen vor. Bei der Herausarbeitung von Anwendungspotentialen sind sowohl methodisch als auch in der Umsetzungsorientierung Schwachstellen erkennbar. Einbezogen in diese These sind auch die unternehmensbezogenen Anwendungen, weil die Förderung von Ökobilanzprojekten in den Unternehmen selbst gleichfalls eine erklärte Zielsetzung der Umweltpolitik ist. Eine Durchsicht der einschlägigen Literatur läßt folgende Schlußfolgerungen zu (7):

- In der staatlichen Umweltpolitik stehen derzeit vergleichende Ökobilanzen im Vordergrund. Die Beteiligung der Akteure ist durchweg gegeben, ein Konsens bei der Bewertung jedoch in der Regel nicht zu erwarten. Ökobilanzbegründete Maßnahmen sind kaum entwickelt (8). Unterentwickelt ist die Optimierungsfunktion von Ökobilanzen. Auch die wichtige Anwendung von Ökobilanzen in der Umsetzung des Kreislaufwirtschaftsgesetzes steht erst am Anfang (9).

- In der Industrie steht die Optimierungsfunktion, weniger der Vergleich im Vordergrund. Anwendungen im Bereich „strategische Planung" in Unternehmen sind bisher wenig belegt. Die hauptsächlichen Anwendungen werden derzeit sogar eher im kommunikativen Bereich und weniger in der Innovation gesehen. Es sind deutliche Widerstände gegen $\mathrm{zu}$ weitgehende methodische Anforderungen festzustellen, demzufolge die betriebliche Anwendungspraxis selten dem „Idealmodell“ nach ISO, sondern eher den spezifischen betrieblichen Realitäten ent- 
spricht. Betriebliche Ökobilanzen sind dabei zu wenig in umfassende betriebliche Entscheidungsprozesse eingebettet.

- Anwendungspotentiale werden auch in neueren Ökobilanzen nur unzureichend berausgearbeitet. Es sei daher die weitergehende These gewagt, daß die nach ISO $14040 \mathrm{zu}$ treffenden „Schlußfolgerungen und Empfehlungen“ als eine bemerkenswerte Schwachstelle der gegenwärtigen Ökobilanz-Praxis zu bezeichnen sind. Demzufolge müssen die Ökobilanz-Ersteller für die systematische Herausarbeitung von Anwendungspotentialen stärker sensibilisiert werden. Als ein große Beachtung verdienendes Positivbeispiel sei die „Ökobilanz Röstkaffee“ genannt, in deren Rahmen es gelungen ist, die Anwendungspotentiale der gesamten Akteurskette im Lebensweg von „Röstkaffee“ systematisch herauszuarbeiten (10). Ein weiteres positives Beispiel ist die zwischenzeitlich sehr gut belegte Anwendung von Ökobilanzen beim Umweltzeichen (11).

These 4: Als besonders konfliktträchtig haben sich vergleichende Ökobilanzen des Umweltbundesamtes mit potentiellen Schlußfolgerungen für die Umweltpolitik herausgestellt, insbesondere hinsichtlich der Frage, was mit den ökologisch schlechter beurteilten Produkten bzw. Systemen geschehen soll.

Ein wichtiges Ziel der staatlichen Umweltpoli$t i k$ ist die Verbesserung der Umweltqualität (des Ökoprofils) von Produkten und Systemen. Ökobilanzen sollen dazu beitragen, ökologisch vorteilhafte Produkte und Systeme zu erkennen. Sie sind eine Basis, diese zum Beispiel durch finanzielle Anreize, Empfehlungen für einen umweltorientierten Einkauf oder die umweltfreundliche Beschaffung zu fördern. Dies gilt auch für die Favorisierung bestimmter Recyclingmaßnahmen im Rahmen des Kreislaufwirtschaftsgesetzes. Damit wird umweltpolitisch gewollter „Druck“ zur Verbesserung der Umweltprofile der in einer Ökobilanz schlechter bewerteten Produkte bzw. Systeme ausgeübt. Es ist jedoch zu betonen, daß eine vergleichende Bewertung nur für die Zeitdauer der bei der Erstellung einer Ökobilanz geltenden Bedingungen gilt und insbesondere im Falle von realisierten Verbesserungen bzw. wesentlichen Veränderungen einer Überprüfung bedarf. Hierbei haben Sensitivitätsanalysen eine große Bedeutung.

\section{Tabelle 1: Beispiele für Ökobilanzprojekte des Umweltbundesamtes (12)}

\begin{tabular}{l} 
Ökobilanz Getränkeverpackungen I \\
\hline Ökobilanz Getränkeverpackungen II \\
\hline Sachbilanz PVC-haltige und \\
PVC-freie Produkte im Bauwesen \\
Ökobilanz Rapsöl
\end{tabular}

Produktlinienanalyse / Ökobilanz

Waschmittel und Waschen

Ökobilanz Papierprodukte

Ökobilanzen in der Kreislaufwirtschaft

Ökobilanz Altölverwertung

Werden in vergleichenden Ökobilanzen Produkte oder Systeme in etwa „ökologisch gleichrangig " bewertet, stehen insbesondere die im Rahmen der Ökobilanz festgestellten und auf unterschiedliche Maßnahmen hinzielenden Verbesserungspotentiale im Mittelpunkt. Der wettbewerbliche Anreiz zur Veranlassung tatsächlicher Maßnahmen besteht hier in der Option, daß bei einer zukünftigen Ökobilanz diese Produkte oder Systeme nicht mehr als gleichrangig angesehen werden können, weil die Verbesserungen sehr unterschiedlich realisiert wurden. Dies gilt auch bei Ökobilanzen, in denen vergleichende Aussagen entweder nicht angestrebt werden oder sich nach Abschluß einer Signifikanzprüfung als nicht belastbar erweisen.

\section{Anmerkung}

(1) Dieser Beitrag fußt auf folgenden Vorträgen:

- "Bilanz und Perspektiven der Nutzung von Ökobilanzen in Politikbewertung und Umweltkennzeichnung" des Works. hops "Ökobilanzen - Trends und Perspektiven" der Gesellschaft Deutscher Chemiker am 26. Juni 1997 in Frankfurt a.M.

- "Ökobilanzen für eine nachhaltige Umweltpolitik" des AK Umweltpolitik der Deutschen Vereinigung für Politische Wissenschaft (DVPW) am 5. Juli 1997 in der FU Berlin

(2) DIN EN ISO 14040 Ökobilanz - Prinzipien und allgemeine Anforderungen, August 1997

(3) Umweltbundesamt (1993): Ökologische Bilanz von Rapsöl bzw. Rapsölmethylester als Ersatz von Dieselkroftstoff (Ökobilanz Rapsöl). Berlin: UBA-TEXTE 4/93

(4) Rubik, Frieder (1997): „Themen, Initiatoren, Methoden und Anwendungen - Wohin bewegen sich die Ökobilanzen". In: FGU (1997): Seminarskript "Produktbezogene Ökobilanzen V“ im Rahmen der UTECH '97. Berlin: Togungsbönde

(5) Umweltbundesamt: Projekt „Bewertung in Ökobilanzen", wird im 1. Quartal 1998 abgeschlossen

6) Grießhammer, Rainer u.a. (1997): Produktlinienanalyse Waschen und Woschmittel. Berlin: UBA-TEXTE 1/1997(Studie des Öko-Instituts im Auftrage des Umweltbundesamts)

(7) Die Schluffolgerungen beruhen ouf folgenden Untersuchungen:

- FTU/VITO/IÖW (1995): Eco-Balances: the uses and limitations of a tool for consultation and for decision-support. on) an Commission he) Milch in der Verpackungsverordnung. erscheint als UBA-TEXTE $97 / 98$. licht), angeführt. werden.

Brüssel (Studie mit Förderung der King Baudouin Foundati-

- Atlantic Consulting, Ernest\&Young, Science Policy Research Unit (1996): The Adoption by Industry of Life Cycle Approaches: Its Implications for Competitiveness, Innovation and Trade. Brüssel: Studie im Auftrag der DG III der Europe-

- Rubik, Frieder; Scholl, Gerd; Grotz, Susanne (1996): Ökologische Entlastungseffekte durch Produktbilanzen. KarIsruhe: Landesanstalt für Umweltschutz Baden-Württemberg - Ankele, Kathrin; Grotz, Susanne; Rubik, Frieder "Standardberichtsbogen II, Fortentwicklung des Konzeptes und Bearbeitung von 30 aktuellen Ökobilanzen" (Studie im Auftrag des Umweltbundesamts, erscheint in der UBA-TEXTE Rei-

(8) Ein aktuelles Beispiel ist die mit den Ergebnissen der Ökobilanz Getrönkeverpackungen I (Schmitz, Stefan U.0. 1995: Ökobilanz für Getrönkeverpackungen. Vergleichende Untersuchung der durch Verpackungssysteme für Frischmilch und Bier hervorgerufenen Umweltbeeinflussungen. Berlin: Umweltbundesamt UBA-Texte 52/95) begründete Einbeziehung des Schlauchbeutels für Milch in die Mehrwegquote bei

(9) Umweltbundesamt: Ökologische Bilanzen in der Abfallwirtschaft anhand der Beispiele Altreifen und Kühlschränke,

(10) Als "Positivbeispiel" wird in diesem Zusammenhang die stark akteursorientierte Ökobilonz "Ökobilanz Röstkoffee - Eine Produktökobilanz von vakuumverpacktem Röstkaffee" im Auftrag von Kraft Jacobs Suchard und dem Umweltsenat Bremen, 1997 (z.Zt. noch nicht veröffent-

(11) Neitzel, Horald (1997): Application of Life Cycle Assessment in Ecolabelling, German Experiences, in "International Journal of Life Cycle Assessment" 1997.

(12) Umweltbundesamt (1997): Materialien zu Ökobilanzen und Lebensweganolysen. Berlin: UBA-TEXTE 26/97; diese Broschüre kann unentgeltlich beim UBA angefordert

\section{Der Autor}

Harald Neitzel ist Diplom-Politologe und leitet das Fachgebiet III 1.3 "Methoden der Produktbewertung, Umweltzeichen" im Umweltbundesamt.

Kontakt: Umweltbundesamt,

Postfach 3300 22, 14191 Berlin, Tel. 030/8903-3703,

Fax.030/8903-3099, e-mail: Harald.Neitzel@uba.de 